\title{
Inteligencia emocional y estrategias de aprendizaje del área de matemática en estudiantes de la Institución Educativa Particular “ Virgen del Rosario”, Huacho - 2018
}

\author{
Emotional intelligence and learning strategies of the area of mathematics in students of \\ the Private Educational Institution "Virgen del Rosario", Huacho - 2018 \\ Henry Cristhian Zubieta Rojas ${ }^{1}$
}

\section{RESUMEN}

Objetivo: Demostrar si existe relación entre la inteligencia emocional y las estrategias de aprendizaje del área de matemática en estudiantes de la Institución Educativa Particular "Virgen del Rosario", Huacho - 2018. Materiales y Métodos: El presente estudio es una investigación de diseño no experimental, transeccional, correlacional. La población está conformada por 57 estudiantes del nivel secundario de la Institución Educativa Particular "Virgen del Rosario", Huacho - 2018. La técnica de recolección de datos fue la Encuesta para ambas variables de estudio y los instrumentos de recolección de datos fueron el inventario de coeficiente emocional de BarOn - ICE y la prueba de Inventario de habilidades y estrategias de aprendizaje elaborado por Weinstein y Colbs. Resultados: El 32\% de los estudiantes encuestados se encuentran en un nivel bajo de inteligencia emocional y bajo de estrategias de aprendizaje del área de matemática, seguido de un $30 \%$ de estudiantes que manifiestan tener un nivel medio de inteligencia emocional y medio de estrategias de aprendizaje del área de matemática. Conclusiones: Existe relación significativa entre la inteligencia emocional y las estrategias de aprendizaje del área de matemática en estudiantes de la Institución Educativa Particular "Virgen del Rosario", Huacho - 2018. Y el grado de correlación es 0.712, es decir, grado de correlación positiva alta.

Palabras clave: Inteligencia emocional, estrategias de aprendizaje, área de matemática

\begin{abstract}
Objective: To demonstrate whether there is a relationship between emotional intelligence and learning strategies in the area of mathematics in students of the Private Educational Institution "Virgen del Rosario", Huacho - 2018. Materials and Methods: The present study is a nonexperimental design research, translational, correlational. The population is made up of 57 students from the secondary level of the Private Educational Institution "Virgen del Rosario", Huacho - 2018. The data collection technique was the Survey for both study variables and the data collection instruments were the inventory of Emotional coefficient of BarOn - ICE and the Inventory of skills and learning strategies test developed by Weinstein and Colbs. Results: $32 \%$ of the students surveyed are in a low level of emotional intelligence and low learning strategies in the area of mathematics, followed by $30 \%$ of students who claim to have a medium level of emotional intelligence and a medium of strategies of Mathematics area learning. Conclusions: There is a significant relationship between emotional intelligence and learning strategies in the area of mathematics in students of the Private Educational Institution "Virgen del Rosario", Huacho - 2018. And the degree of correlation is 0.712 , that is, degree of positive correlation high
\end{abstract}

Keywords: cerebral hemisphere; stimulation program; hemisphere right; hemisphere left.

\section{INTRODUCCIÓN}

\section{Inteligencia emocional}

En el año 1997, Barón plantea una definición de inteligencia emocional como el conjunto de destrezas y habilidades emocionales, sociales y personales, que impactan en la capacidad que se tiene para adaptarse y enfrentar las presiones del entorno. Por tanto, esta inteligencia es relevante y de gran relevancia en la consecución del éxito en la vida así también en la salud y bienestar emocional (Ugarriza, 2001).

Por lo tanto, para ser exitosos en la vida y alcanzar la realización profesional y personal, es necesario desarrollar la inteligencia, pero no solo la inteligencia cognitiva, que tienen que ver con los conocimientos que se tengan y las habilidades y destrezas desarrolladas en una determinada actividad o varias, sino que a ello se tienen que tener en cuenta la inteligencia emocional, es decir, la capacidad de los individuos para conducir sus emociones de la mejor manera y la de los demás. Porque los estudios han comprobado que un adecuado manejo de la inteligencia emocional garantiza el bienestar general de las personas y la salud emocional que es la base para proponerse las metas más altas y lograrlas.

\section{Áreas de la inteligencia emocional}

Para un mejor estudio de la Inteligencia Emocional, se ha descompuesto está en componentes, los mismos que permiten medir que tan buena inteligencia emocional se posee. Son cinco: Componente intrapersonal, componente interpersonal, adaptabilidad, manejo del estrés y estado de ánimo general.

\section{a) Componente intrapersonal}

Este componente refiere a la capacidad de comprender los sentimientos propios, las emociones, establecer diferentes, saber percatarse de las que muestran los demás y la razón por la cual surgen estos. Asimismo, comprender la habilidad para expresar sentimientos, emociones y creencias desde un modo empático, esto es, sin dañar al prójimo, hacer valer los derechos de uno mismo, autoconocerse lo positivo y lo negativo, lo posible y lo limitante y las valoración a uno mismo (Guarniz, 2017, p. 33).

Este componente desarrolla la habilidad de conocer nuestros sentimientos y emociones y exteriorizarlos de manera adecuada. Asimismo, permite defender nuestras ideas y manera de pensar sin herir a los demás sino compartiendo con respeto lo que se piensa y en lo que se cree, pero también siendo asequible con lo que piensan y creen los demás.

\section{b) Componente interpersonal}

Este componente se refiere a la habilidad que se tiene para considerar, comprender y percatarse empáticamente de la actitud de las personas, además de conservar y establecer interacciones con los demás de manera satisfactoria. Asimismo, incluye a aquella capacidad de mostrarse como una persona que apoya y sirve de elemento constructivo de un grupo social (Guarniz, 2017, p. 36).

\footnotetext{
1 Maestría en Docencia Superior e Investigación Universitaria. Escuela de Posgrado. UNJFSC.

Recibido: 18/01/2019 Aprobado: 26/03/2019
} 
Este componente permite que las personas desarrollen habilidades para llevarse bien con los demás dentro de un clima de respeto y consideración, ser considerado conos demás entendiendo sus diferencias y ser parte de un grupo donde desarrolla valores como la responsabilidad compartida, la lealtad y el compañerismo, entre otros valores fundamentales que permiten a las personas interrelacionarse entre ellas.

\section{c) Manejo del estrés}

Esta referido al actuar de manera tolerante y controlada de los impulsos o por situaciones de estrés, frente a ello mostrar calma pese a la presión existente y sabiendo equilibrar situaciones de estrés (Guarniz, 2017, p. 41).

Este componente permite a las personas afrontar problemas y dificultades de la vida diaria y saber elegir las mejores decisiones $y$, sobre todo, de manera oportuna para que estos problemas no terminen agobiando y por el contrario se responda a las diferentes situaciones con calma y prontitud en la solución. No es fácil desarrollar esta habilidad, y la mayoría de personas terminan en episodios mayores de problemas, por eso la importancia de conocerlo y ponerlo en práctica.

\section{d) Estado de ánimo}

Se refiere a la habilidad propia del ser humano para mostrarse feliz con su vida, regocijo propio y por los demás, transmitir sentimiento positivos, asumir actitud positiva y mantener en todo momento una postura firme pese a situaciones tóxicas o sentimiento negativos (Guarniz, 2017, p. 42).

Este componente tiene que ver con la forma como ven las personas la vida, a pesar de que no siempre se pasa por buenos momentos, sin embargo, el optimismo y el estado de tanimo no se debe malograr. Al contrario, es necesario mantenerlo para hacer llevadero aquellas situaciones que presentan y que son difíciles.

\section{e) Adaptabilidad}

La dimensión adaptabilidad se relaciona con la capacidad humana para adaptarse a nuevas situaciones de manera eficiente y a valorar idóneamente las mismas. Incluye, además, la creación de soluciones a problemas de naturaleza cotidiana (Guarniz, 2017, p. 39).

Este componente permite a las personas desarrollar la toma de conciencia de que la vida tiene matices que van hacer que algunas veces se esté tranquilo y en otras ocasiones se afronten dificultades, situaciones nuevas, retos, riesgos, etc., pero que a todo ello se tiene ir acostumbrando la persona e ir ajustando sus emociones de acuerdo a cada cosa nueva que va viviendo.

\section{Aprendizaje de la matemática.}

Respecto a este aprendizaje, Lazaro (2012), señala que es de tipo estructural, sobre todo al referirse a la adquisición de los conceptos, ya que se sostiene que aprender es alterar construcciones o estructura de modo global (p. 37).

El aprendizaje de la matemática es especial, porque prepara al individuo en la resolución de problemas, la aplicación del razonamiento y la lógica a eventos de la vida cotidiana, etc. $Y$ lo hace cada vez más fácil y más expectante la vida de todos.

\section{Estrategias de aprendizaje.}

Son un conto de procedimientos que utiliza el estudiante a fin de lograr un aprendizaje eficaz para que se adquiera, se relacione, se procese, se retenga, se recupere y se regule la información (Guarniz, 2017, p. 57)
Barios plantea que las estrategias de aprendizaje son una de las luces que orienta la investigación en el campo educativo, ya que facilitan la adquisición, el desarrollo y la conducción de procesos que ayuden a adquirir contenidos para facilitar un aprendizaje eficaz (Peña y Cosi, 2014, p. 27).

Por lo tanto, el docente debe manejar bien y dominar las estrategias que va utilizar para propiciar aprendizajes en sus estudiantes dentro de un ambiente propicio para que se de este aprendizaje y con conocimiento de que está utilizando los mejores medios y materiales que ayudan a los estudiantes aun cuando el tema tiene dificultades para aprenderse.

\section{Elementos de las estrategias de aprendizaje}

\section{a) Adquisición de información}

Son estrategias que brindan orientación o control de la atención optimizando el proceso de repetición de la información (Guarniz, 2017, p. 59)

Están dirigidas a recoger los conocimientos nuevos a través de una serie de actividades que permiten que a partir de los saberes previos se produzcan nuevos. Esta actividad es importante y se da al inicio de todo proceso de aprendizaje.

\section{b) Codificación de información}

Se encargan de la puesta en acción de los procesos codificadores, a través de la traducción a un código de información adquirido que sea almacenado en la memoria de largo plazo (Guarniz, 2017, p. 62).

Una vez que se ha producido la adquisición del conocimiento en la etapa anterior, aquí lo que se hace es un 'proceso mental a través del cual se ordena la información en el cerebro de las personas para que tenga significancia y sirva para que el individuo tenga mayor capacidad en algún tema específico.

\section{c) Recuperación de la información}

Estas estrategias sirven para apoyar la búsqueda de información que se encuentra en la memoria a largo plazo y su vez permitir su recuperación (Guarniz, 2017, p. 63).

Este elemento se da cuando durante la vida profesional o personal tenemos que hacer uso de los conocimientos que poseemos para ponerlos en práctica y desarrollar alguna actividad relacionada con el mismo.

\section{d) Apoyo de la información}

Son encargadas de apoyar el procesamiento de la información a fin de hacer más eficaz la adquisición, la codificación y la recuperación, tal que se incremente la motivación, la autoestima y la atención (Guarniz, 2017, p. 65).

Este elemento es importante y viene a completar el proceso de aprendizaje, ya que tiene que ver con la motivación que se debe generar en los estudiantes para mantener el interés por el estudio.

\section{MATERIAL Y MÉTODOS}

El diseño del estudio es no experimental, transeccional, correlacional. La población consta de 57 estudiantes del nivel secundario de la Institución Educativa Particular "Virgen del Rosario", Huacho - 2018. La técnica para recolectar datos fue la encuesta en el caso de ambas variables de estudio: Inteligencia emocional y Estrategias de aprendizaje del área de matemática.

Los instrumentos de recolección de datos fueron el inventario de coeficiente emocional de BarOn - ICE y la prueba de Inventario de habilidades y estrategias de aprendizaje 
elaborado por Weinstein y Colbs para la medición del grado en que los estudiantes utilizan estrategias y métodos de aprendizaje y estudio.

\section{RESULTADOS Y DISCUSIÓN}

\section{Tabla 1}

Coeficiente de Correlación de Pearson de la variable Inteligencia emocional y la variable Estrategias de aprendizaje del área de matemática

\begin{tabular}{|c|c|c|c|}
\hline \multicolumn{4}{|c|}{ Correlaciones } \\
\hline & & $\begin{array}{l}\text { Inteligencia } \\
\text { emocional }\end{array}$ & $\begin{array}{c}\text { Estrategias de } \\
\text { aprendizaje } \\
\text { del área de } \\
\text { matematica }\end{array}$ \\
\hline \multirow{3}{*}{$\begin{array}{l}\text { Inteligencia } \\
\text { emocional }\end{array}$} & $\begin{array}{c}\text { Correlación de } \\
\text { Perarson }\end{array}$ & 1 & $712^{\star *}$ \\
\hline & Sig. & & 0,000 \\
\hline & $\begin{array}{c}\text { (bilateral) } \\
\mathrm{N}\end{array}$ & 30 & 30 \\
\hline \multirow{3}{*}{$\begin{array}{l}\text { Estrategias } \\
\quad \text { de } \\
\text { aprendizaje } \\
\text { del área de } \\
\text { matemática }\end{array}$} & $\begin{array}{l}\text { Correlación de } \\
\text { Pearson }\end{array}$ & $712^{* *}$ & 1 \\
\hline & Sig & 0,000 & \\
\hline & $\begin{array}{c}\text { (bilateral) } \\
\mathrm{N}\end{array}$ & 30 & 30 \\
\hline
\end{tabular}

Interpretación: La Tabla 1 muestra los resultados del Coeficiente de Correlación de Pearson que da como resultado un nivel de significancia 0,000 (menor al p-valor $=0,05$ ), resultados que dan suficiente evidencia para aceptar la hipótesis alterna y rechazar la hipótesis nula de investigación, es decir, existe relación significativa entre la inteligencia emocional y las estrategias de aprendizaje del área de matemática en estudiantes de la Institución Educativa Particular "Virgen del Rosario", Huacho - 2018, y el grado de correlación es 0,712 , es decir, grado de correlación positiva alta.

\section{Tabla 2}

Coeficiente de Correlación de Pearson de la dimensión Componente intrapersonal y la variable Estrategias de aprendizaje del área de matemática

\begin{tabular}{|c|c|c|c|}
\hline \multicolumn{4}{|c|}{ Correlaciones } \\
\hline & & $\begin{array}{l}\text { Componente } \\
\text { intrapersonal }\end{array}$ & $\begin{array}{c}\text { Estrategias de } \\
\text { aprendizaje } \\
\text { del área de } \\
\text { matemática }\end{array}$ \\
\hline \multirow{3}{*}{$\begin{array}{l}\text { Componte } \\
\text { interpersonal }\end{array}$} & $\begin{array}{l}\text { Correlación } \\
\text { de Pearson }\end{array}$ & 1 & $643^{* *}$ \\
\hline & $\begin{array}{c}\text { Sig. } \\
\text { (bilateral) }\end{array}$ & & 0,000 \\
\hline & $\mathrm{N}$ & 30 & 30 \\
\hline \multirow{3}{*}{$\begin{array}{l}\text { Estratégias } \\
\text { de } \\
\text { aprendizaje } \\
\text { del área de } \\
\text { matemática }\end{array}$} & $\begin{array}{l}\text { Correlación } \\
\text { de Pearson }\end{array}$ & $643^{* * *}$ & 1 \\
\hline & $\begin{array}{c}\text { Sig. } \\
\text { (bilateral) }\end{array}$ & 0,000 & \\
\hline & $\mathrm{N}$ & 30 & 30 \\
\hline
\end{tabular}

Interpretación: La Tabla 2 muestra los resultados del Coeficiente de Correlación de Pearson que da como resultado un nivel de significancia 0,000 (menor al p-valor $=0,05$ ), resultados que dan suficiente evidencia para aceptar la hipótesis alterna y rechazar la hipótesis nula de investigación, es decir, existe relación significativa entre el componente intrapersonal y las estrategias de aprendizaje del área de matemática en estudiantes de la Institución Educativa Particular "Virgen del Rosario", Huacho - 2018, y el grado de correlación es 0,643 , es decir, grado de correlación positiva moderada.

Tabla 3

Coeficiente de Correlación de Pearson de la dimensión Componente interpersonal y la variable Estrategias de aprendizaje del área de matemática

\begin{tabular}{|c|c|c|c|}
\hline \multicolumn{4}{|c|}{ Correlaciones } \\
\hline & & $\begin{array}{l}\text { Componente } \\
\text { interpersonal }\end{array}$ & $\begin{array}{c}\text { Estratégias de } \\
\text { aprendizaje } \\
\text { del área de } \\
\text { matemática }\end{array}$ \\
\hline \multirow{3}{*}{$\begin{array}{l}\text { Componente } \\
\text { interpersonal }\end{array}$} & $\begin{array}{l}\text { Correlación } \\
\text { de Pearson }\end{array}$ & 1 & $600^{* *}$ \\
\hline & $\begin{array}{c}\text { Sig. } \\
\text { (bilateral) }\end{array}$ & & 0,000 \\
\hline & $\mathrm{N}$ & 30 & 30 \\
\hline \multirow{3}{*}{$\begin{array}{l}\text { Estrategias de } \\
\text { aprendizaje } \\
\text { del área de } \\
\text { matemática }\end{array}$} & $\begin{array}{l}\text { Correlación } \\
\text { de Pearson }\end{array}$ & $600^{* *}$ & 1 \\
\hline & $\begin{array}{c}\text { Sig. } \\
\text { (bilateral) }\end{array}$ & 0,000 & \\
\hline & $\mathrm{N}$ & 30 & 30 \\
\hline
\end{tabular}

Interpretación: La Tabla 3 muestra los resultados del Coeficiente de Correlación de Pearson que da como resultado un nivel de significancia 0,000 (menor al p-valor $=0,05$ ), resultados que dan suficiente evidencia para aceptar la hipótesis alterna y rechazar la hipótesis nula de investigación, es decir, existe relación significativa entre el componente interpersonal y las estrategias de aprendizaje del área de matemática en estudiantes de la Institución Educativa Particular "Virgen del Rosario", Huacho - 2018, y el grado de correlación es 0,600 , es decir, grado de correlación positiva moderada.

\section{Tabla 4}

Coeficiente de Correlación de Pearson de la dimensión manejo del estrés y la variable Estrategias de aprendizaje del área de matemática.

\begin{tabular}{|c|c|c|c|}
\hline \multicolumn{4}{|c|}{ Correlaciones } \\
\hline & & $\begin{array}{l}\text { Manejo de } \\
\text { estrés }\end{array}$ & $\begin{array}{c}\text { Estrategias de } \\
\text { aprendizaje } \\
\text { del área de } \\
\text { matemática }\end{array}$ \\
\hline \multirow{3}{*}{$\begin{array}{c}\text { Componte } \\
\text { interpersonal }\end{array}$} & $\begin{array}{l}\text { Correlación } \\
\text { de Pearson }\end{array}$ & 1 & $643^{* *}$ \\
\hline & Sig. (bilateral) & & 0,000 \\
\hline & $\mathrm{N}$ & 30 & 30 \\
\hline \multirow{3}{*}{$\begin{array}{l}\text { Estratégias de } \\
\text { aprendizaje } \\
\text { del área de } \\
\text { matemática }\end{array}$} & $\begin{array}{l}\text { Correlación } \\
\text { de Pearson }\end{array}$ & $643^{* * *}$ & 1 \\
\hline & Sig. (bilateral) & 0,000 & \\
\hline & $\mathrm{N}$ & 30 & 30 \\
\hline
\end{tabular}

Interpretación: La Tabla 4 muestra los resultados del Coeficiente de Correlación de Pearson que da como resultado un nivel de significancia 0,000 (menor al p-valor $=0,05$ ), resultados que dan suficiente evidencia para aceptar la hipótesis alterna y rechazar la hipótesis nula de investigación, es decir, existe relación significativa entre el manejo del estrés y las estrategias de aprendizaje del área de matemática en 
estudiantes de la Institución Educativa Particular "Virgen del Rosario", Huacho - 2018, y el grado de correlación es 0,643, es decir, grado de correlación positiva moderada.

\section{Tabla 5}

Coeficiente de Correlación de Pearson de la dimensión Estados de ánimo y la variable Estrategias de aprendizaje del área de matemática

\begin{tabular}{|c|c|c|c|}
\hline \multicolumn{4}{|c|}{ Correlaciones } \\
\hline & & $\begin{array}{l}\text { Estado de } \\
\text { ánimo }\end{array}$ & $\begin{array}{c}\text { Estratégias de } \\
\text { aprendizaje } \\
\text { del área de } \\
\text { matemática }\end{array}$ \\
\hline \multirow{3}{*}{$\begin{array}{l}\text { Componente } \\
\text { interpersonal }\end{array}$} & $\begin{array}{l}\text { Correlación } \\
\text { de Pearson }\end{array}$ & 1 & $401^{* *}$ \\
\hline & $\begin{array}{c}\text { Sig. } \\
\text { (bilateral) }\end{array}$ & & 0,028 \\
\hline & $\mathrm{N}$ & 30 & 30 \\
\hline \multirow{3}{*}{$\begin{array}{l}\text { Estrategias } \\
\text { de } \\
\text { aprendizaje } \\
\text { del área de } \\
\text { matemática }\end{array}$} & $\begin{array}{l}\text { Correlación } \\
\text { de Pearson }\end{array}$ & $401^{*}$ & 1 \\
\hline & $\begin{array}{c}\text { Sig. } \\
\text { (bilateral) }\end{array}$ & 0,028 & \\
\hline & $\mathrm{N}$ & 30 & 30 \\
\hline
\end{tabular}

Interpretación: La Tabla 5 muestra los resultados del Coeficiente de Correlación de Pearson que da como resultado un nivel de significancia 0,028 (menor al p-valor $=0,05$ ), resultados que dan suficiente evidencia para aceptar la hipótesis alterna y rechazar la hipótesis nula de investigación, es decir, existe relación significativa entre el estado de ánimo y las estrategias de aprendizaje del área de matemática en estudiantes de la Institución Educativa Particular "Virgen del Rosario", Huacho - 2018, y el grado de correlación es 0,401, es decir, grado de correlación positiva moderada

\section{Tabla 6}

Coeficiente de Correlación de Pearson de la dimensión Adaptabilidad y la variable Estrategias de aprendizaje del área de matemática

\begin{tabular}{|c|c|c|c|}
\hline \multicolumn{4}{|c|}{ Correlaciones } \\
\hline & & Adaptabilidad & $\begin{array}{c}\text { Estrategias de } \\
\text { aprendizaje } \\
\text { del área de } \\
\text { matemática }\end{array}$ \\
\hline \multirow{3}{*}{$\begin{array}{l}\text { Componte } \\
\text { interpersonal }\end{array}$} & $\begin{array}{l}\text { Correlación } \\
\text { de Pearson }\end{array}$ & 1 & $367^{*}$ \\
\hline & $\begin{array}{c}\text { Sig. } \\
\text { (bilateral) }\end{array}$ & & 0,046 \\
\hline & $\mathrm{N}$ & 30 & 30 \\
\hline \multirow{3}{*}{$\begin{array}{l}\text { Estratégias } \\
\text { de } \\
\text { aprendizaje } \\
\text { del área de } \\
\text { matemática }\end{array}$} & $\begin{array}{l}\text { Correlación } \\
\text { de Pearson }\end{array}$ & $367^{* \star *}$ & 1 \\
\hline & $\begin{array}{c}\text { Sig. } \\
\text { (bilateral) }\end{array}$ & 0,046 & \\
\hline & $\mathrm{N}$ & 30 & 30 \\
\hline
\end{tabular}

Interpretación: La Tabla 6 muestra los resultados del Coeficiente de Correlación de Pearson que da como resultado un nivel de significancia 0,046 (menor al p-valor $=0,05$ ), resultados que dan suficiente evidencia para aceptar la hipótesis alterna y rechazar la hipótesis nula de investigación, es decir, existe relación significativa entre la adaptabilidad y las estrategias de aprendizaje del área de matemática en estudiantes de la Institución Educativa Particular "Virgen del
Rosario", Huacho - 2018 y el grado de correlación es 0,367 , es decir, grado de correlación positiva baja

\section{CONCLUSIONES}

Existe relación significativa entre la inteligencia emocional y las estrategias de aprendizaje del área de matemática en estudiantes de la Institución Educativa Particular "Virgen del Rosario", Huacho - 2018 con un nivel de significancia de 0,000 y el grado de correlación es positiva alta $(0,712)$.

Existe relación significativa entre la componente intrapersonal y las estrategias de aprendizaje del área de matemática en estudiantes de la Institución Educativa Particular "Virgen del Rosario", Huacho - 2018 con un nivel de significancia de 0,000 , y el grado de correlación es positiva moderada $(0,643)$.

Existe relación significativa entre el componente interpersonal y las estrategias de aprendizaje del área de matemática en estudiantes de la Institución Educativa Particular "Virgen del Rosario", Huacho - 2018 con un nivel de significancia de 0,000 y el grado de correlación es positiva moderada $(0,600)$.

Existe relación significativa entre el manejo del estrés y las estrategias de aprendizaje del área de matemática en estudiantes de la Institución Educativa Particular "Virgen del Rosario", Huacho - 2018 con un nivel de significancia de 0,000 , y el grado de correlación es positiva moderada $(0,643)$.

Hay relación significativa entre el estado de ánimo y las estrategias de aprendizaje del área de matemática en estudiantes de la Institución Educativa Particular "Virgen del Rosario", Huacho - 2018 con un nivel de significancia de 0,028 , y el grado de correlación es positiva moderada $(0,401)$.

Hay relación significativa entre la adaptabilidad y las estrategias de aprendizaje del área de matemática en estudiantes de la Institución Educativa Particular "Virgen del Rosario", Huacho - 2018 con un nivel de significancia de 0,046, y el grado de correlación es positiva baja $(0,367)$.

\section{RECOMENDACIONES}

Se debe capacitar a los docentes en estrategias de aprendizaje que se relacionen con el estado emocional de los estudiantes para lograr su interés y despertar motivación por la asignatura.

Desarrollar talleres de autoconocimiento y autorrealización entre los estudiantes para mejorar su componente intrapersonal.

Llevar a cabo actividades de integración entre los estudiantes para mejorar el componente interpersonal de cada uno de ellos.

Desarrollar técnicas de manejo del estrés para mejorar la salud emocional de los estudiantes y lograr mejores aprendizajes.

Promover actividades que propicie estados de ánimo adecuados em los estudiantes y mejorar las estrategias aplicadas a los estudiantes para que se sientan animados al aprendizaje y no se desanimen.

Realizar técnicas de adaptabilidad para que los estudiantes logren afrontar situaciones diversas y se adapten a ellas. 


\section{REFERENCIAS BIBLIOGRÁFICAS}

Casabianca, D. (2015). La influencia de la inteligencia emocional en las habilidades matemáticas de tipo numérico operativo en los estudiantes que inician la educación superior en la Universidad Sergio Arboleda. Universidad Sergio Arboleda. Bogotá, Colombia: USERGIOARBOLEDA. Obtenido de https://repository.usergioarboleda.edu.co/bitstream/ handle/11232/847/La\%20influencia $\% 20 \mathrm{de} \% 20$ la $\% 20$ inteligencia $\% 20$ emocional $\% 20$ en $\% 20$ las $\% 20$ habilidades $\% 20$ matem $\%$ C3\%A1ticas. $\% 20$ Universidad \% 20 Sergio $\% 20$ Arboleda. pdf?sequence $=2 \&$ isAllowed=y

Guarniz, L. (2017). Inteligencia emocional, motivación y estrategias de aprendizaje en estudiantes de III ciclo de Enfermería. Tesis de Maestría, Universidad César Vallejo, Lima, Perú. Obtenido de http://repositorio. ucv.edu.pe/bitstream/handle/UCV/7737/Guarniz_ CLE.pdf?sequence=1\&isAllowed=y

Huanca, E. (2012). Niveles de inteligencia emocional de docentes de una Institución Educativa del distrito de Ventanilla - Callao. Universidad San Ignacio de Loyola, Escuela de Postgrado. Lima, Perú: USIL. Obtenido de http://repositorio.usil. edu.pe/bitstream/123456789/1173/1/2012 Huanca_Niveles $\% 20$ de $\% 20$ inteligencia $\% 20$ emocional\%20de $\% 20$ docentes $\% 20$ de $\% 20$ una $\% 20$ instituci \% C 3\%B 3n \% 20 educativa $\% 20$ del $\% 20$ distrito\%20de\%20Ventanilla-\%20Callao.pdf
Lázaro, D. (2012). Estrategias didácticas y aprendizaje de la matemática en el programa de estudios por experiencia laboral. Universidad San Martín de Porres, Instituto para la calidad de la EducaciónSección de Posgrado. Lima, Perú: USMP. Obtenido de http://www.repositorioacademico.usmp.edu.pe/ bitstream/usmp/613/3/lazaro_db.pdf

Peña, C., \& Cosi, E. (2014). Relación entre la inteligencia emocional y las estrategias de aprendizaje. PESQUIMAT Revista de la Facultad de Ciencias Matemáticas de la Universidad Nacional Mayor de San Marcos, XVII(1), 25-37. Obtenido de http:// revistasinvestigacion.unmsm.edu.pe/index.php/ matema/article/view/12504/11187

Stover, J., Uriel, F., \& Fernández, M. (2012). Inventario de Estrategias de Aprendizaje y Estudio: Análisis Psicométricos de una Versión Abreviada. (U. N. Córdoba, Ed.) Revista Argentina de Ciencias del Comportamiento, 4(3), 4-12. doi:http://dx.doi. org/10.30882/1852.4206.v4.n3.5162

Ugarriza, N. (2001). La evaluación de la inteligencia emocional através del inventario de BarOn (I-CE) en una muestra de Lima Metropolitana. (U. d. Lima, Ed.) Persona 4, 129-160. Obtenido de https://www. redalyc.org/html/1471/147118178005/ 